\section{Hydrodynamics of planetary nebulae}

Vincent Icke

Sterrewacht Leiden, The Netherlands

\section{Stellar evolution}

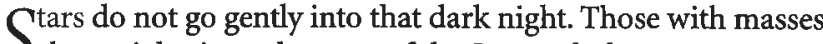
$S$ above eight times the mass of the Sun explode as supernovae, outshining for a few weeks their parent galaxy. But even lowmass stars make a show of their departure. Because they are so much more numerous than the heavies, the remains of defunct solar-type stars litter the galactic landscape. Known as planetary nebulae, they appear so frequently that many of them are found quite nearby; with apparent sizes of many minutes of arc, these are excellent objects for viewing with an amateur telescope. The moniker 'planetary' has nothing to do with planets; it means 'planet-like', because an emission line of doubly ionised oxygen gives these nebulae a greenish glow, reminiscent of a planetary disk.

Stars shine due to the nuclear fusion of hydrogen, producing helium, carbon, and heavier elements. The central temperature in low-mass stars is too low to produce much beyond the CNOgroup. When the hydrogen in the core is exhausted-which takes about 10 billion years for the Sun-the core contracts. The temperature is, as in Wien's Law, inversely proportional to a length, in this case the core radius. The luminosity is proportional to the fourth power of the temperature and the second power of the radius, so that the core increases a hundredfold in brightness for every factor 10 it contracts. Thus, when the core contracts, the stellar atmosphere is heated from below and puffs up, ultimately reaching a size comparable with the orbit of Venus. The luminosity of the cooling core is radiated away through this enormous surface, so that the effective temperature of the star is low, of the order of $2000 \mathrm{~K}$ or even less. In its final ten million years, the star has become a red giant.

During the initial stages of this process, some fusion still occurs in a shell around the core, but this state is overstable. Ultimately, thermal pulses of increasing amplitude drive off the outer shell, leaving behind a naked core with a surface temperature up to several hundred thousand kelvin. Radiation pressure from this brilliant pinpoint, soon to cool down and become a white dwarf, drives a supersonic wind into the departing outer shell. It is the collision between these two winds-a dense shell at tens of kilometres per second, hit by a tenuous gas at a few thousand $\mathrm{km} / \mathrm{s}$ - that produces the beautiful planetary nebulae.

\section{Colliding winds}

Originally, it was thought that most PNs are spherical (Fig.1, left). After all, that is what their parent stars are likely to be, and the physics of the formation of such shells was well understood. The outer 'slow' wind expands with a velocity of about $20 \mathrm{~km} / \mathrm{s}$ and a density of $10^{10}$ atoms per $\mathrm{m} 3$. The inner 'fast' wind races outward with speeds up to $2000 \mathrm{~km} / \mathrm{s}$, and a much lower density, typically $10^{7}$. The interacting winds produce a typical shock/contact-discontinuity/shock configuration. Seen from the central star, and following a radial flow line, the gas is very supersonic, and moves outward with a Mach number of 40-100. Then the gas passes through a strong shock, which converts most of the kinet-

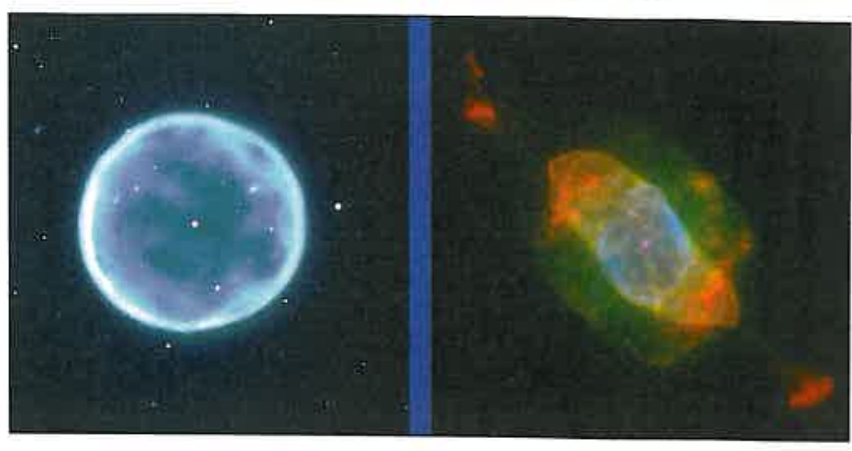

$\triangle$ Fig. 1: Left:the spherical planetary nebula Abell 39;image courtesy of G. Jacoby and Kitt Peak National Observatory. Right: the bipolar NGC7009, observed by B. Balick with WFPC2 on the Hubble Space Telescope.

ic into thermal energy. This creates a thick layer of very high temperature and pressure, which pushes against the 'slow' gas at a contact discontinuity where the gas density jumps upward but the pressure and the velocity are continuous. Further out, the slow gas runs into whatever external medium there is, and a second shock is formed.

Using standard hydrodynamic conservation laws, these configurations were readily analysed (e.g. Kwok 1982), and compared well with the observations of spherical PNs. The fast wind is almost unobservable, and the region beyond the inner shock, having a temperature in the millions of kelvin, is properly observed only in X-rays, which has only recently become possible with the advent of satellites that can observe X-rays with large collecting area. The shocked slow wind, which forms the archetypical 'planetary nebula', stands out clearly in the light of various emission lines, most notably $\mathrm{H} \alpha,\left[\mathrm{N}^{+}\right]$and $\left[\mathrm{O}^{2+}\right]$. The nebular spectrum is dominated by the reprocessed ultraviolet radiation of the central white dwarf.

\section{Kompaneyets revisited}

But the spherical textbook cases turned out to be a minority (Fig. 1, right). Many planetaries even have an extreme dumbbell shape (Fig.2; for detailed reviews, see Schwarz 1993 or Sahai 2002). In 1987 Bruce Balick (University of Washington, Seattle) published a classification scheme in which, for the first time, the whole range of shapes was taken seriously, all the way from purely spherical (Fig.1) to completely double-lobed (Fig.2). In addition, Balick (1987) supposed that the shaping of bipolar nebulae was due to a hypothetical aspherical shape of the outer slow shell, even though a mechanism for producing this was-and is-unknown.

When Balick visited Sterrewacht Leiden on a sabbatical leave, he asked me to think about the hydrodynamics of interacting

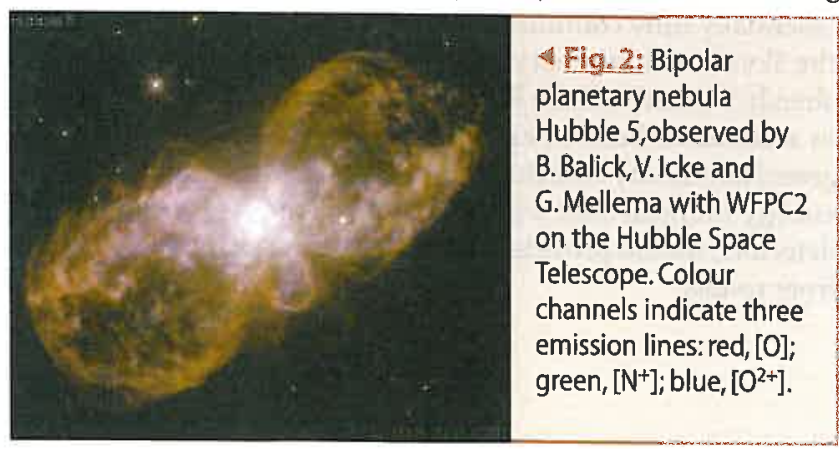




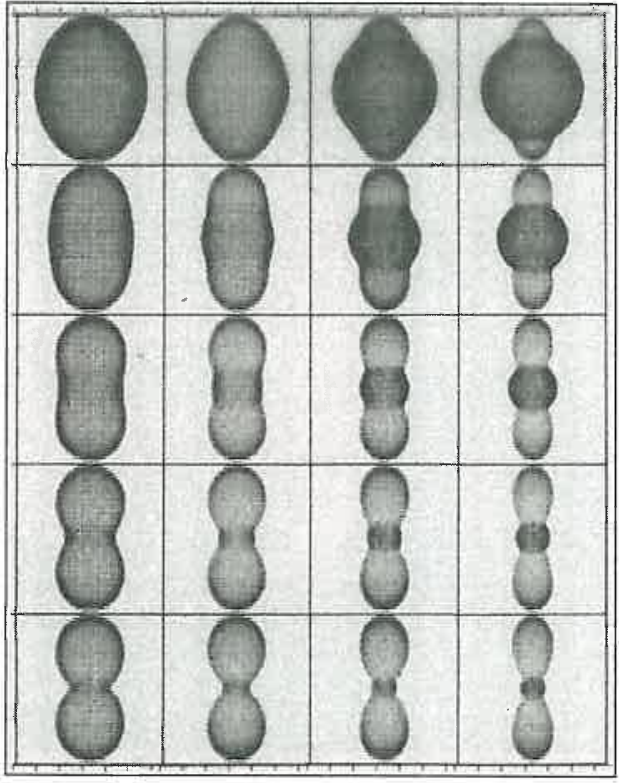

4. Fig. 3: Analytical models for bipolarplanetary nebulae. From left to right, the pole/equator density contrast increases; from top to bottom, time increases. Images have been scaled to fit the boxes.

aspherical winds. The main problem, as I saw it, was finding a suitable approximation for the propagation of a very strong shock through an inhomogeneous atmosphere. Of course I knew the elegant analytical solution that Kompaneyets (1960) had found for the evolution of a strong shock in an exponential atmosphere, and I formulated the PN problem in the same approximation. The shape $r(\theta, t)$ of the outer shock can be found analytically from the equation

$$
\frac{\partial r}{\partial t}=\left\{\frac{(\gamma+1) P}{2 \rho}\left[1+\left(\frac{1}{r} \frac{\partial r}{\partial \theta}\right)^{2}\right]\right\}^{1 / 2}
$$

Here $P$ is the pressure behind and $\rho$ the gas density ahead of the shock. In the PN case, the density $\rho$ in the outer atmosphere is far from exponential. Instead, it is cylindrically symmetric, with a distribution dictated by the flow of the slow wind. The velocity of this gas is almost constant, so that the density can be approxi-

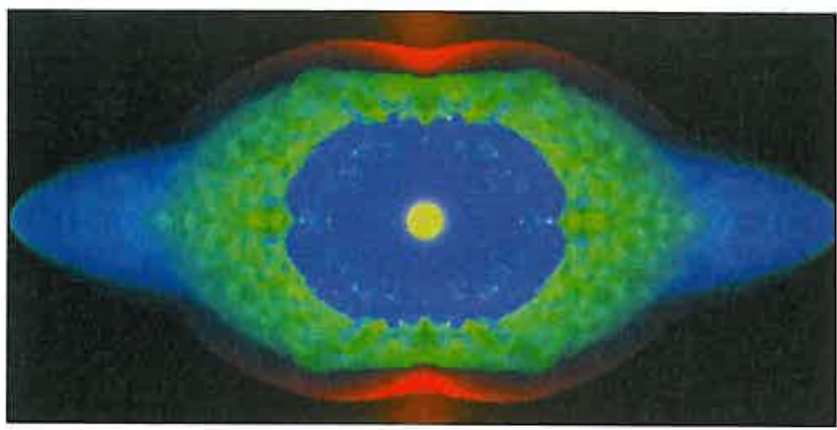

Fig. 4: Snapshot of adiabatic PN model.Symmetry axis horizontal, equatorial plane vertical. Red is the density, green temperature, blue the absolute value of the velocity. mated by $K(\theta) / r^{2}$, where $K$ is an arbitrary function of the polar angle $\theta$ and $r$ is the radial distance to the star. To my surprise and delight, I discovered that this equation can be solved exactly by separation of variables. The solution is

$$
\rho(r, \theta)=r^{-2} K(\theta) ; \log r=E t-\int \sqrt{E^{2} K(\theta)-1} d \theta+g(E)
$$

with separation constant $E$ and arbitrary function $g(E)$. This form allows the easy generation of predicted shock shapes for a great variety of confining density distributions (Fig.3).

The sequence of bipolar shapes generated by this analytical solution matched Balick's classification scheme almost perfectly (Balick 1987; Icke 1991, and references therein).

\section{Numerical hydrodynamics}

But the analytic solution said nothing about what is perhaps the most intriguing part of a PN, namely its interior. To solve that part of the flow, numerical hydrodynamics was necessary. The difficulty here is, that the gigantic jumps in pressure and density in PNs present severe difficulties to ordinary, diffusive numerical methods (Soker \& Livio 1988). Using my 'LCD' variant of Boris \& Book's robust flux-corrected transport code, I managed to keep the diffusive effects of numerical hydrodynamics within acceptable bounds (Fig.4).

Subsequent work by Mellema and co-workers, using Roe's approximate Riemann solver, greatly refined these results. Finally, Mellema completed the picture by including radiative transfer and ionisation effects in the equations of motion (Mellema 1995 and references therein).

In the early investigations of bipolar nebulae, the hydrodynamical models concentrated on disk-wind interactions that are 'energy driven'. A central star blows a spherical wind into a toroidal nebula; the wind has such a low density and high speed that the surrounding (inner) shock generates a very hot, pressure dominated layer that then drives the outer shock. Because flow in the high pressure region is very subsonic, inhomogeneities in

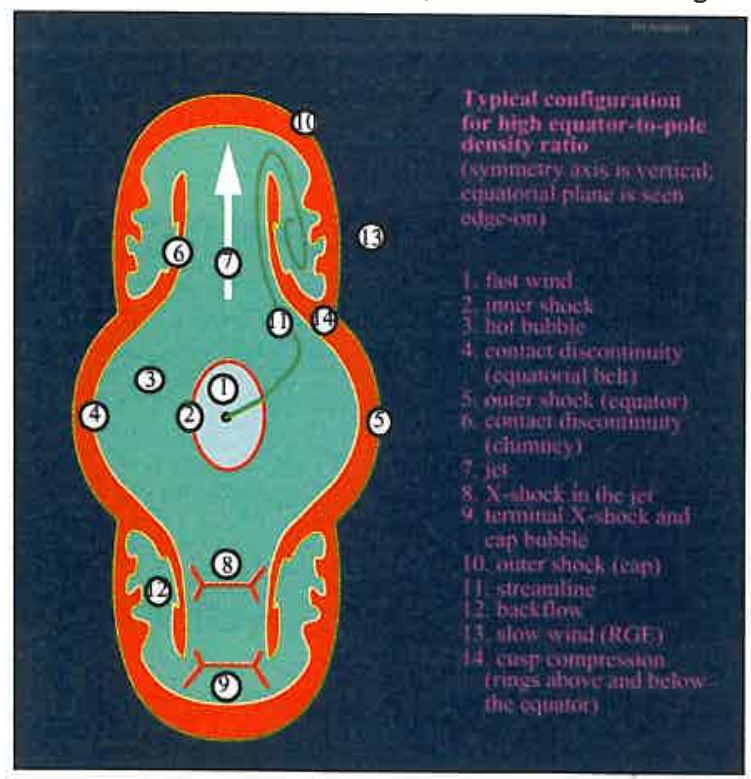

A Fig. 5 : the Identification of features discovered in numerical models for bipolar planetary nebulae. Most of these have now been identified in observed images. 
this layer are mostly smoothed out, so that the outer shock and the nebular shape are well rounded.

These computations showed a wealth of structures in the interior of the PN (Fig.5), most of which were subsequently identified in observed images, especially those obtained at the European Southern Observatory and with the Hubble Space Telescope. It was even discovered that disk-wind interactions can produce sharply collimated supersonic jets (Icke et al. 1992).

\section{Cooling flows}

If the flow is strongly compressible, a thick high-pressure smoothing shell does not develop. This regime is often called 'momentum driven'. In such flows the gas still has considerable momentum after passage through the inner shock, and thereby shapes the outer nebula by direct impact rather than by a mediating pressure. Strong cooling can provide the requisite compressibility, and instabilities and other interesting effects will certainly occur. In order to allow dimensionless scaling of the simulations, the high compressibility can be mimicked by picking a small value of the Poisson constant (usually $\gamma=1.1$ ). A typical result is shown in Figure 6. When these computations are used to generate images as they would be observed, the similarity with actual nebulae is particularly striking (Fig.7).

It is expected that stars of higher mass (in the range 2-8 solar masses) produce nebulae that do not cool quickly, and are therefore 'energy driven', whereas the stars that have smaller masses produce the highly compressible, 'momentum driven' PNs. This prediction remains to be verified.

\section{Apotheosis: the Red Rectangle}

Astronomy is great, if only because there are always objects that look so strange that they seem to defy explanation. One of these is the 'Red Rectangle' (Icke 1981 and references therein), a nebula looking just like what its name says on the Palomar plates on which it was discovered. New observations (Van Winckel, priv.comm.) clearly show that the Red Rectangle flow is biconical, but it also seems to pulsate : the stream is intermittent, which at first sight would be very difficult to produce in the interaction between a steady spherical outflow and a stationary surrounding disk. However, the central star is a binary, which could modulate the flow.

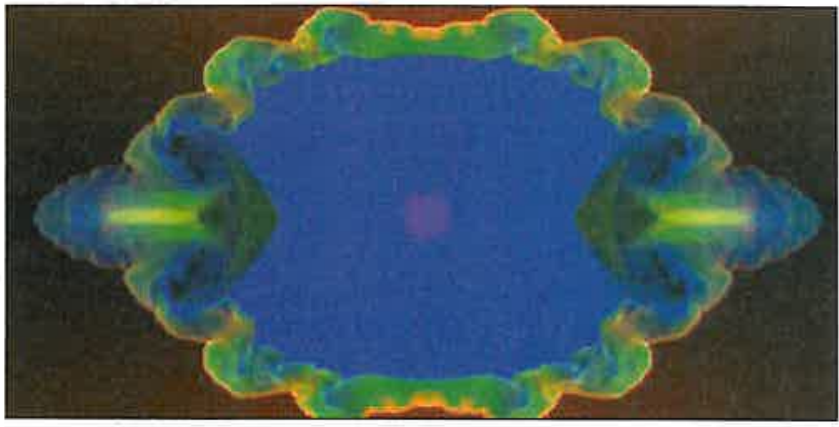

4ig. 6: Snapshot of a highly compressible PN model (pseudocooling). All parameters are the same as in Figure 4 , except that the value of the adiabatic index is 1.1 instead of 5/3. Symmetry axis horizontal, equatorial plane vertical. Red is the density, green the temperature, blue the absolute value of the velocity. Notice the collapsed shock-contact configuration, and the instabilities of the outer shock front.

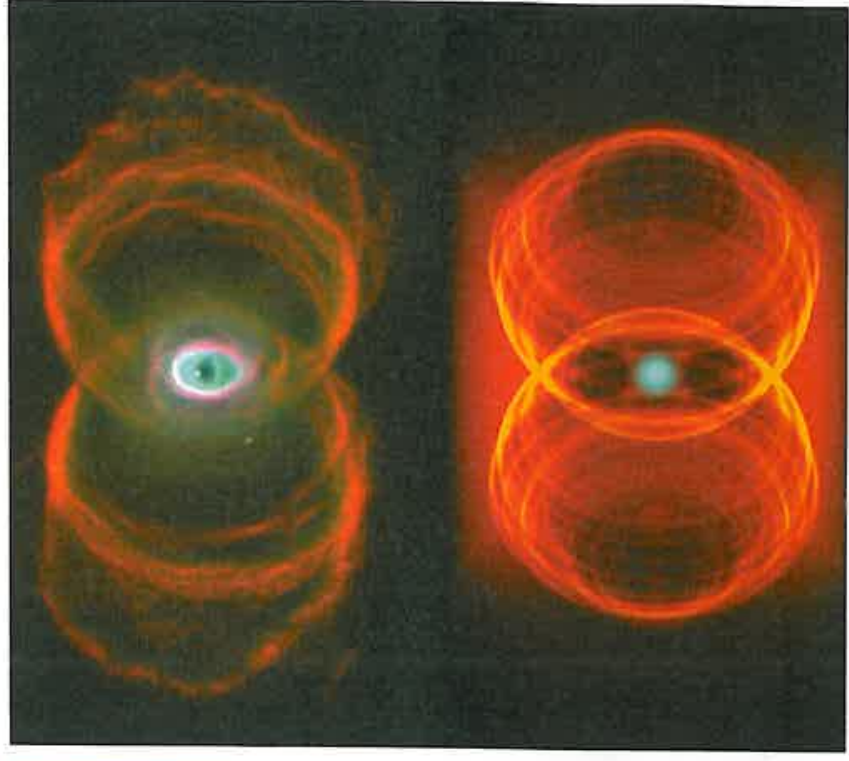

A Fig. 7: Bipolar planetary nebula MyCn18, as observed by R. Sahai \& J.Trauger with WFPC2 on the Hubble Space Telescope, compared with hydrodynamic pseudo-cooling model.

In the case of momentum-driven flow, the instabilities that occur in the inner flow regime make the outflow far from stationary. In some cases, the gas is focused into a double cone, when it passes through the aspherical inner shock (cf. Fig.5). The density of the Red Rectangle is so high, and the central star(s) so cool, that radiative losses will be considerable, and highly compressible 'momentum driven' outflow is almost guaranteed. Focusing of the primary gas through the inner shock may create a biconical outflow pattern that can produce the characteristic X-shape of the outer nebula.

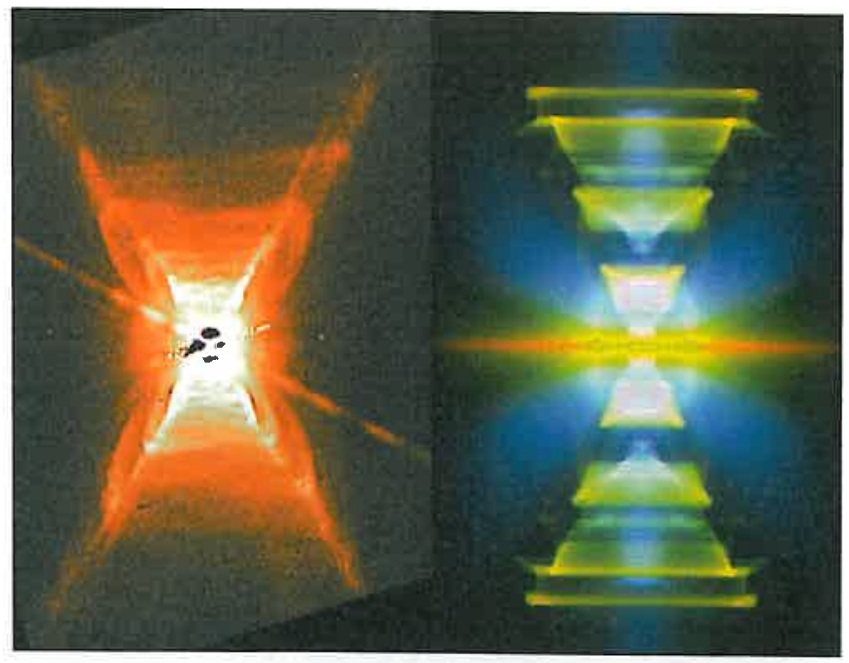

Alg. 8: Left: Observed image of the Red Rectangle, courtesy of Van Winckel. Right: Pseudo-observation of a hydrodynamic simulation with $\gamma=1.1$. Symmetry axis vertical, equatorial plane horizontal. The image was produced by computing the scattering of white light in the density field produced by the hydrocode. Note the 'Mach stems' at the place where the recurving bow shocks intersect on the symmetry axis. 
Observations such as those by Van Winckel can thus be modelled in great detail. Computational experiments show that a specific set of features is common to a wide range of compressible biconical flows. Key features are (Fig.8): (1) cup-shaped density ridges close to the central source, which become ragged rings at higher altitude; (2) the recurving bow shocks ahead of the gaseous rings that are shot upwards from the centre; (3) intersecting shocks on the symmetry axis, forming Mach stems ('X-shocks'); (4) the gradual widening of the effective cone opening angle at higher altitudes, due to the outward pressure behind these shocks. One feature that I initially thought to be problematic for my interpretation, namely the occurrence of $\mathrm{X}$-shocks on the axis, turned out to be present on closer inspection of Van Winckel's $\mathrm{H} \alpha$ image.

\section{Conclusions}

Bipolar nebulae are due to an interaction between two winds: a slow, dense outflow deposited during the time immediately after the red giant phase, and a very fast, tenuous wind driven by radiation from the dying white dwarf. Analytical and numerical calculations allow the identification of almost all flow features. Notable exceptions are the so-called 'FLIERs' (fast, low-ionisation emission regions). These many forms of mass loss fertilise the interstellar plasma with the materials of life, and show the admirable ways in which low-mass stars rage against the dying of their light.

\section{References}

Full colour-coded versions of some of the flow patterns shown here will be kept as long as disk space permits on www.strw.LeidenUniv.nl/ 176icke/, from which they can be downloaded. QuickTime movies of some of my simulations can be obtained on request from icke@strw.LeidenUniv.nl .

[1] Balick, B. 1987 Astron.J. 94,671

[2] Icke, V. 1981 Astrophys.J. 247, 152

[3] Icke, V. 1991 Astron.Astrophys 251, 369

[4] Icke, V., Mellema, G., Balick, B., Eulderink, F., \& Frank, A.: 1992 Nature 355, 524

[5] Kompaneyets, A.S. (1960). Doklady Akad. Nauk SSSR 130, 1001

[6] Kwok, S. 1982 Astrophys.J. 258, 280

[7] Mellema, G., 1995 Mon.Not.Roy.astron.Soc. 277, 173

[8] Sahai, R., 2002 Rev. Mex. Astron. Astrophys. 13, 133

[9] Schwarz, H.E., p.223 in: Mass loss on the AGB and beyond, H.E. Schwarz (Ed.), ESO Conf.Wrksh.Proc. 46 (1993)

[10] Soker, N., \& Livio, M. 1989 Astrophys.J. 339, 268
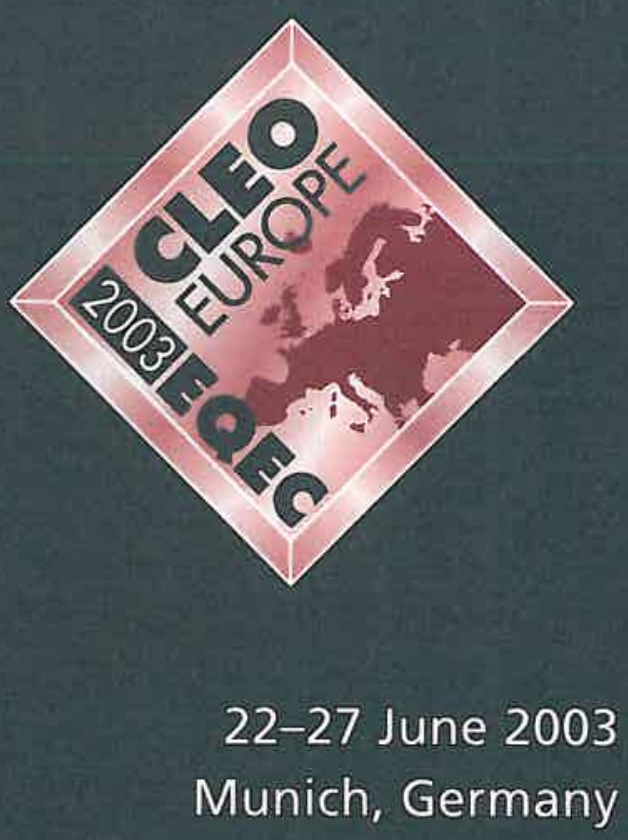

Conference Secretariat

European Physical Society BP 2136, F=68050 Mulhouse Cedex, France tel: $+333893294.42 \mathrm{fax}:+3338932.94 .49$ email:eps.conf@uhafi web: wwwiceceuroperorg
CLEO/Europe-EQEC 2003 is Europe's premier joint conference on lasers, electro-optics and fundamental quantum electronics.

The conference will be held at the Munich International Conference Centre in conjunction with Laser 2003, the largest European exhibition of laser and electro-optic equipmentand services

CLEO Europe-EQEC 2003 is the place to discover the latest research developments, applications, and technology in the field.

\section{Early registration has already started}

\section{Save $15 \%$ by registering now}

For online registration and more information visit

www.cleoeurope.org 